\title{
The Use of Micro-Raman Spectroscopy to Monitor High-Pressure High- Temperature Annealing of Ion-Implanted GaN Films
}

\author{
M. Kuball ${ }^{1}$, J.M. Hayes ${ }^{1}$, T. Suski ${ }^{2}$, J. Jun ${ }^{2}$, H.H. Tan ${ }^{3}$, J.S. Williams ${ }^{3}$, and \\ C. Jagadish ${ }^{3}$ \\ ${ }^{1}$ H.H. Wills Physics Laboratory, University of Bristol, Bristol BS8 1TL, UK \\ ${ }^{2}$ UNIPRESS, Polish Academy of Sciences, Solowska 29, 01-142 Warsaw, Poland \\ ${ }^{3}$ Department of Electronic Materials and Engineering, Research School of Physical \\ Sciences and Engineering, The Australian National University, Canberra, ACT 0200, \\ Australia
}

\begin{abstract}
We have investigated the high-pressure high-temperature annealing of $\mathrm{Mg} / \mathrm{P}$ implanted GaN films using visible and ultraviolet (UV) micro-Raman spectroscopy. The results illustrate the use of Raman spectroscopy to monitor processing of $\mathrm{GaN}$ where fast feedback is required. The structural quality and the stress in ion-implanted GaN films was monitored in a 40nm-thin surface layer of the sample as well as averaged over the sample layer thickness. We find the nearly full recovery of the crystalline quality of ionimplanted $\mathrm{GaN}$ films after annealing at $1400-1500^{\circ} \mathrm{C}$ under nitrogen overpressures of $1.5 \mathrm{GPa}$. No significant degradation effects occurred in the GaN surface layer during the annealing. The high nitrogen overpressures proved very effective in preventing the nitrogen out-diffusion from the GaN surface. Stress introduced during the annealing was monitored. Raman spectra of ion-implanted GaN films were investigated at different temperatures and excitation wavelengths to study the GaN phonon density of states.
\end{abstract}

\section{INTRODUCTION}

The family of III-V nitrides (GaN, InGaN, AlGaN) has recently attracted great interest because of their wide spectrum of applications ranging from opto-electronic devices for the blue-ultraviolet spectral region [1] to high-temperature electronic devices [2]. A large number of processing steps is required for the III-V nitride device fabrication, for example, reactive ion etching (RIE) or focused ion beam (FIB) etching [3], annealing to achieve a low contact resistance to n-type and p-type GaN [4], annealing to activate the Mg-acceptors for $\mathrm{p}$-doping [5]. The non-invasive monitoring of the processing of III-V nitrides is of great interest to gain good control over the III-V nitride device fabrication. In this paper, we demonstrate the use of Raman scattering to monitor non-invasively the processing of $\mathrm{GaN}$, illustrated on the example of the high-pressure high-temperature annealing of ion-implanted $\mathrm{GaN}$ films. Ion-implantation is highly attractive for the integration of III-V nitride devices into circuits, however, introduces severe lattice damage [6]. Annealing at temperatures in excess of $1300^{\circ} \mathrm{C}$ is needed to recover the crystalline quality of ion-implanted GaN films. AlN cap layers [7] or highpressure nitrogen atmospheres [8,9] have to be employed to prevent the GaN surface decomposition at such high temperatures. Raman scattering provides information on the vibrational states of $\mathrm{GaN}$, which track noninvasively the crystalline quality and the stress in ion-implanted GaN films. The crystalline quality was determined from the $\mathrm{E}_{2}$ phonon 
linewidth of $\mathrm{GaN}$, whilst the stress from the $\mathrm{E}_{2}$ phonon frequency [10]. We restrict this paper on the monitoring of the crystalline quality and the stress in GaN. Results on the free carrier concentration in ion-implanted GaN films monitored by Raman scattering will be reported elsewhere. High optical efficiency micro-Raman systems nowadays make the recording of Raman spectra with very short integration times possible. Fast feedback on the material properties of III-V nitride layers using Raman scattering is therefore possible.

\section{EXPERIMENT}

Visible and ultraviolet (UV) micro-Raman spectra were recorded from the top surface of annealed Mg/P-implanted GaN films in backscattering Z(X,.) $\underline{Z}$ geometry, i.e., unpolarized detection, with $1-2 \mu \mathrm{m}$ spatial resolution using a visible and an ultraviolet (UV) Renishaw micro-Raman system with the 514nm- and 488nm-line of an $\mathrm{Ar}^{+}$-laser and with the $325 \mathrm{~nm}$-line of a HeCd-laser as excitation source, respectively. The spectral resolution of the Raman setup was $2-3 \mathrm{~cm}^{-1}$ for all excitation wavelengths used. The GaN films used for this study were $1.5 \mu \mathrm{m}$ thick, grown by metalorganic chemical vapor deposition (MOCVD) on sapphire (0001) substrates (commercially available - CREE). $\mathrm{Mg}$ - and $\mathrm{P}$-ions were implanted into the $\mathrm{GaN}$ by a multistep process of increasing the ion energy up to $1 \mathrm{MeV}$. As a result, a uniform (across the whole layer thickness)

concentration of $\mathrm{Mg}$ and $\mathrm{P}$ equivalent to $2 \times 10^{19} \mathrm{~cm}^{-3}$ was achieved [9]. The samples were annealed at temperatures ranging from $1200^{\circ} \mathrm{C}$ to $1500^{\circ} \mathrm{C}$ in a high-pressure furnace. Pressures of 1-1.5GPa were applied, with purified $\mathrm{N}_{2}$ as the pressure-transmitting medium. Mg pieces were placed in the high-pressure furnace near the sample to provide a magnesium overpressure during the annealing. Un-implanted GaN films (taken from the same wafer as used for the ion-implantation) were annealed under the same conditions as the ion-implanted $\mathrm{GaN}$ films for comparison.

\section{RESULTS AND DISCUSSION}

Figure 1 displays the $\mathrm{E}_{2}$ phonon linewidth of ion-implanted and of un-implanted $\mathrm{GaN}$ films obtained (a) under $514 \mathrm{~nm}$ - and (b) under $325 \mathrm{~nm}$-excitation as function of the annealing temperature. The $\mathrm{E}_{2}$ linewidth is a measure for the crystalline quality of the $\mathrm{GaN}$. The results obtained under 514nm-excitation (Figure 1(a)) probe the crystalline quality of the GaN films averaged over the sample layer thickness, those obtained under $325 \mathrm{~nm}$-excitation (Figure 1(b)) determine the crystalline quality in a sample surface layer of $1 /(2 \alpha)=40 \mathrm{~nm}$ thickness due to the absorption of the laser light in the $\mathrm{GaN}(\alpha=$ absorption coefficient at $325 \mathrm{~nm}$ taken from [11]). The $\mathrm{E}_{2}$ linewidth of ion-implanted $\mathrm{GaN}$ films decreases with increasing annealing temperature. After $1500^{\circ} \mathrm{C}$ anneals, it is comparable to the $\mathrm{E}_{2}$ linewidth of as-grown $\mathrm{GaN}$ films. No broadening of the $\mathrm{E}_{2}$ phonon linewidth was detectable for annealing temperatures as high as $1500^{\circ} \mathrm{C}$ under $325 \mathrm{~nm}$ excitation. No significant surface degradation occurred therefore during the annealing. For un-implanted GaN films, high-pressure high-temperature annealing results in a decrease of the $\mathrm{E}_{2}$ linewidth for annealing temperatures of $1300-1400^{\circ} \mathrm{C}$, however, this is reversed at $1500^{\circ} \mathrm{C}$. High-pressure high-temperature annealing restores the crystalline 

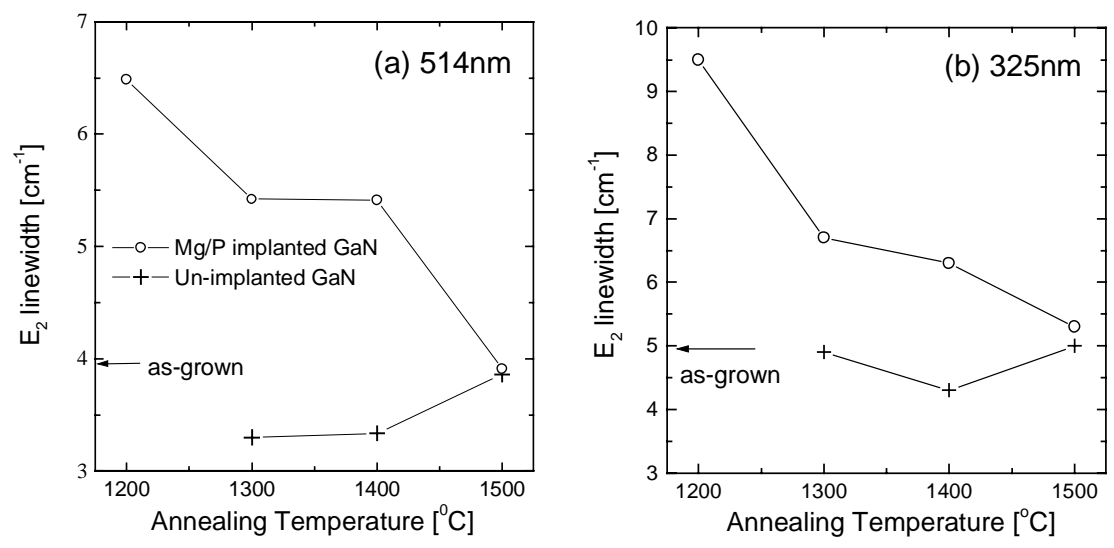

Figure 1. $E_{2}$ phonon linewidth of ion-implanted and of un-implanted GaN films determined (a) under $514 \mathrm{~nm}$ - and $(b)$ under $325 \mathrm{~nm}$-excitation. The $E_{2}$ phonon linewidth is a measure for the crystalline quality of the GaN.

quality of ion-implanted $\mathrm{GaN}$ films to a large extent. It also improves the crystalline quality of un-implanted GaN films.

The homogeneity of the investigated samples can be estimated by comparing the $\mathrm{E}_{2}$ linewidths determined under 514nm- and 325nm-excitation (Figure 1(a) and (b)). Ionimplanted and un-implanted $\mathrm{GaN}$ films annealed at temperatures in excess of $1300^{\circ} \mathrm{C}$ exhibit a more or less homogeneous crystalline quality throughout the layer thickness. The $\mathrm{E}_{2}$ linewidths determined under $514 \mathrm{~nm}$ - and under $325 \mathrm{~nm}$-excitation agree within the experimental resolution. In contrast to the above, a reduced surface crystalline quality is found in ion-implanted $\mathrm{GaN}$ films annealed at $1200^{\circ} \mathrm{C}$ with an increased $\mathrm{E}_{2}$ linewidth under $325 \mathrm{~nm}$-excitation.

Figure 2 displays the $\mathrm{E}_{2}$ phonon frequency of ion-implanted and of un-implanted $\mathrm{GaN}$ films obtained (a) under $514 \mathrm{~nm}$ - and (b) under $325 \mathrm{~nm}$-excitation. The $\mathrm{E}_{2}$ frequency is a measure for the stress in the GaN. Compressive stress emerges and shifts the $\mathrm{E}_{2}$ phonon to larger wavenumbers with increasing annealing temperature. After the $1500^{\circ} \mathrm{C}$ anneal, a frequency shift of $3.9 \mathrm{~cm}^{-1}$ and of $1.5 \mathrm{~cm}^{-1}$ is found under $514 \mathrm{~nm}$-excitation for ion-implanted and for un-implanted $\mathrm{GaN}$ films, respectively, with respect to the sample before ion-implantation and annealing. For ion-implanted $\mathrm{GaN}$ films, the $\mathrm{E}_{2}$ frequencies obtained under $514 \mathrm{~nm}$ - and under $325 \mathrm{~nm}$-excitation agree within the experimental resolution. The stress is more or less homogeneous throughout the layer thickness. Inhomogeneous stress is found in un-implanted $\mathrm{GaN}$ films annealed at $1500^{\circ} \mathrm{C}$ with an increased $\mathrm{E}_{2}$ frequency under $325 \mathrm{~nm}$-excitation.

Figure 3 compares Raman spectra of as-implanted, un-annealed GaN films recorded under $488 \mathrm{~nm}$-excitation at different temperatures. For comparison results obtained under $325 \mathrm{~nm}$-excitation at room temperature are also shown. Ion-implantation gives rise to a high-density network of defects [6] lifting the wavevector conservation of the Raman scattering process (disorder-induced Raman scattering). The Raman spectrum is closely related to the $\mathrm{GaN}$ phonon density of states for non-resonant excitation conditions [12], for example, for an excitation wavelength of $488 \mathrm{~nm}$ far below the $\mathrm{GaN}$ 

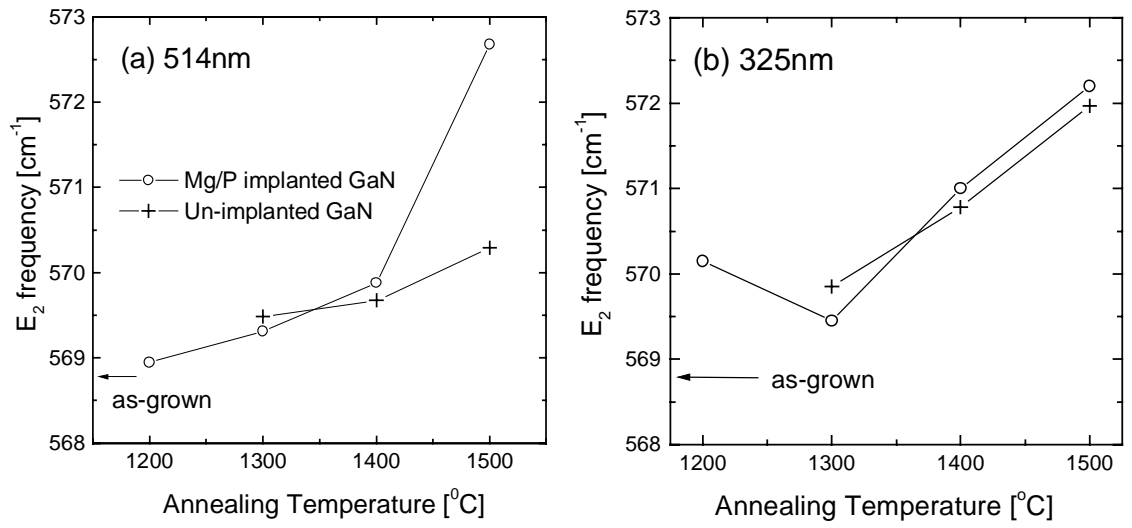

Figure 2. $E_{2}$ phonon frequency of ion-implanted and of un-implanted GaN films as function of the annealing temperature determined (a) under $514 \mathrm{~nm}$ - and $(b)$ under $325 n m$-excitation. The $E_{2}$ phonon frequency is a measure for the stress in the GaN.

bandgap. The corresponding Raman spectrum recorded at room temperature contains three contributions: a peak at $574 \mathrm{~cm}^{-1}$, at $668 \mathrm{~cm}^{-1}$ and at $716 \mathrm{~cm}^{-1}$. Their linewidth decreases with decreasing temperature, their frequency increases by $1-2 \mathrm{~cm}^{-1}$ from $300 \mathrm{~K}$ to $10 \mathrm{~K}$. This temperature-induced frequency shift is in good agreement with values reported for the $\mathrm{E}_{2}$ and the $\mathrm{A}_{1}(\mathrm{LO})$ phonons of $\mathrm{GaN}$ [13]. Theoretical results on the $\mathrm{GaN}$ phonon density of states from Nipko et al. [14] calculated using a rigid-ion lattice dynamical model are displayed in Figure 3. We find good agreement between the Raman spectra recorded under $488 \mathrm{~nm}$-excitation and the calculated phonon density of states, except for the Raman peak at $668 \mathrm{~cm}^{-1}$, which can be attributed to local vibration modes [15]. Resonance effects can distort the GaN phonon density of states in the Raman spectrum [12]. This is illustrated in Figure 3 for $325 \mathrm{~nm}$-excitation. The UV excitation close to the GaN bandgap resonantly enhances the Raman peak located at $721 \mathrm{~cm}^{-1}$ related to the GaN LO phonons. The UV excitation does neither enhance the local vibrational mode at $668 \mathrm{~cm}^{-1}$ nor the Raman peak at $574 \mathrm{~cm}^{-1}$.

Micro-Raman scattering allowed us to monitor non-invasively the processing of $\mathrm{GaN}$, namely, to track the crystalline quality and the stress in ion-implanted and in unimplanted $\mathrm{GaN}$ films after high-pressure high-temperature annealing. Using different excitation wavelengths $\mathrm{GaN}$ properties were probed in the sample surface layer as well as averaged over the sample layer thickness to estimate the homogeneity of the stress and the crystalline quality in the investigated samples. The main result of significance is the nearly full recovery of the crystalline quality of ion-implanted GaN films after highpressure high-temperature annealing at $1400-1500^{\circ} \mathrm{C}$ tracked by the decreasing $\mathrm{E}_{2}$ phonon linewidth in Figure 1. Improvements in the crystalline quality were also achieved for un-implanted $\mathrm{GaN}$ films by annealing at $1300-1400^{\circ} \mathrm{C}$. UV Raman scattering found no significant surface degradation effects during the annealing at temperatures as high as $1500^{\circ} \mathrm{C}$. The high nitrogen overpressures proved very effective in preventing the surface decomposition which would normally occur at a temperature of $1000-1200^{\circ} \mathrm{C}[16,17]$. Stress is introduced into the GaN layers during the annealing (Figure 2) and shifts the $\mathrm{E}_{2}$ 


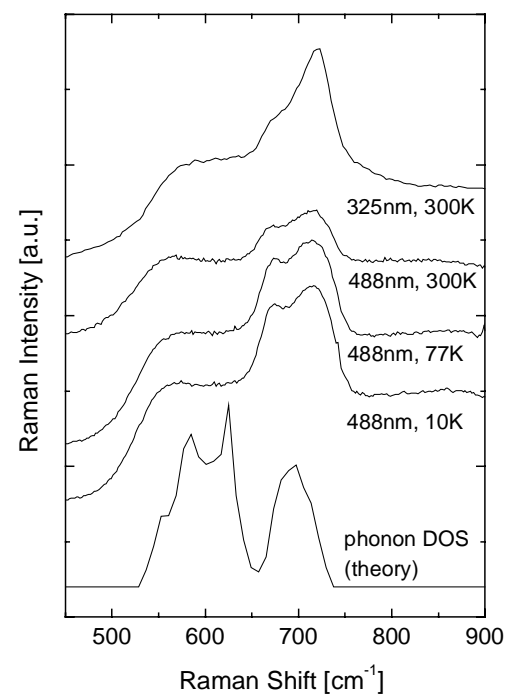

Figure 3. Raman spectra of as-implanted, un-annealed GaN films recorded under 488nm-excitation at different temperatures and recorded under $325 \mathrm{~nm}$-excitation at room temperature. The theoretical phonon density of states of GaN is from [14].

phonon frequency to higher wavenumbers with $2.9 \mathrm{~cm}^{-1} / \mathrm{GPa}$ [10]. Annealing at 1300$1500^{\circ} \mathrm{C}$ therefore introduces a compressive stress of $0.3-0.5 \mathrm{GPa}$ in un-implanted $\mathrm{GaN}$ films (Figure 2(a)). The increased crystalline quality, i.e., the decreased defect density, in annealed un-implanted GaN films (Figure 1(a)) reduces their ability to relax the stress, which arises from the large lattice mismatch between $\mathrm{GaN}$ and the sapphire substrate. Stress also arises from the inclusion of impurities into the $\mathrm{GaN}$ crystal lattice such as the $\mathrm{Mg}$ and $\mathrm{P}$ in the ion-implanted GaN films (Figure 2(a)).

\section{CONCLUSIONS}

We have investigated the high-pressure high-temperature annealing of ionimplanted and of un-implanted GaN films using micro-Raman spectroscopy. Using ultraviolet (UV) and visible excitation wavelengths the crystalline quality and the stress was monitored in the sample surface layer as well as averaged over the sample layer thickness, respectively. Disorder-induced Raman scattering was used to investigate the $\mathrm{GaN}$ phonon density of states. The results illustrate the use of Raman spectroscopy to monitor processing of $\mathrm{GaN}$ where fast feedback is required.

\section{ACKNOWLEDGEMENTS}

We acknowledge financial support for the work in Bristol by Renishaw plc (Dr. G.D. Pitt), by EPSRC (grant no. GR/M15590) and by the Royal Society (grant no. 
20141). J.M.H. is supported by a CASE studentship from DERA Malvern (Dr. T. Martin). We thank C.K. Loong (Argonne National Laboratory) for providing the theoretical data on the $\mathrm{GaN}$ phonon density of states.

\section{REFERENCES}

[1] S. Nakamura, M. Senoh, S. Nagahama, N. Iwasa, T. Yamada, T. Matsushita, H. Kiyoku, Y. Sugimoto, T. Kozaki, H. Umemoto, M. Sano, and K. Chocho, Appl. Phys. Lett. 72, 211 (1998).

[2] S. Yoshida and J. Suzuki, Jpn. J. Appl. Phys. 37, L482 (1998).

[3] I. Adesida, C. Youtsey, A.T. Ping, F. Khan, L.T. Romano, and G. Bulman, MRS Internet J. Nitride Semiconductor Res. 4S1, G1.4 (1999); M. Kuball, F.H. Morrissey, M. Benyoucef, I. Harrison, D. Korakakis, and C.T. Foxon, Phys. Stat. Sol. (a) 176, 355 (1999).

[4] F. Ren, in GaN and Related Material, Optoelectronic properties of semiconductors and superlattices, Vol. 2, edited by M.O. Manasreh (Gordon and Breach Science Publishers, Amsterdam, 1997), pp. 433-469.

[5] S. Nakamura, T. Mukai, M. Senoh, and N. Iwasa, Jpn. J. Appl. Phys. 31, L139 (1992).

[6] J.C. Zolper, H.H. Tan, J.S. Williams, J. Zou, D.J.H. Cockayne, S.J. Pearton, M. Hagerott Crawford, and R.F. Karlicek, Jr., Appl. Phys. Lett. 70, 2729 (1997); H.H. Tan, J.S. Williams, J. Zou, D.J.H. Cockayne, S.J. Pearton, J.C. Zolper, and R.A. Stall, Appl. Phys. Lett. 72, 1190 (1998).

[7] X.A. Cao, C.R. Abernathy, R.K. Singh, S.J. Pearton, M. Fu, V. Sarvepalli, J.A. Sekhar, J.C. Zolper, D.J. Rieger, J. Han, T.J. Drummond, R.J. Shul, and R.G. Wilson, Appl. Phys. Lett. 73, 229 (1998).

[8] T. Suski, J. Jun, M. Leszcyński, H. Teisseyre, S. Strite, A. Rockett, A. Pelzmann, M. Kamp, amd K.J. Ebeling, J. Appl. Phys. 84, 1155 (1998).

[9] T. Suski, J. Jun, M. Leszczynski, H. Teisseyre, I. Gryzegory, S. Porowski, J.M. Baranowski, A. Rocket, S. Strite, A. Stonert, A. Turos, H.H. Tan, J.S. Williams, and C. Jagadish, Mat. Res. Soc. Symp. Proc. 492, 949 (1998).

[10] F. Demangeot, J. Frandon, M.A. Renucci, O. Briot, B. Gil, R.-L. Aulombard, MRS Internet J. Nitride Semicond. Res. 1, 23 (1996).

[11] J.F. Muth, J.H. Lee, I.K. Shmagin, R.M. Kolbas, H.C. Casey, Jr., B.P. Keller, U.K. Mishra, and S.P. DenBaars, Appl. Phys. Lett., 71, 2572 (1997).

[12] M. Cardona in Light Scattering in Solids II, edited by M. Cardona and G. Güntherodt (Springer, Heidelberg, 1982), pp. 19-178.

[13] M.S. Liu, L.A. Bursill, S. Prawer, K.W. Nugent, Y.Z. Tong, G.Y. Zhang, Appl. Phys. Lett. 74, 3125 (1999).

[14] J.C. Nipko, C.-K. Loong, C.M. Balkas, and R.F. Davis, Appl. Phys. Lett. 73, 34 (1998).

[15] W. Limmer, W. Ritter, R. Sauer, B. Menschling, C. Liu, and B. Rauschenbach, Appl. Phys. Lett. 72, 2589 (1998).

[16] M. Kuball, F. Demangeot, J. Frandon, M.A. Renucci, J. Massies, N. Grandjean, R.L. Aulombard, and O. Briot, Appl. Phys. Lett. 73, 960 (1998).

[17] J.M. Hayes, M. Kuball, A. Bell. I. Harrison, D. Korakakis, and C.T. Foxon, Appl. Phys. Lett. 75, 2097 (1999). 